\title{
Publisher Correction: Maya meteor mystery
}

May Chiao

Correction to: Nature Astronomy https://doi.org/10.1038/s41550-017-0234-x (2017); published online 1 September 2017.

In the version of this Research Highlight originally published, the figure credit was incorrect. The figure has now been correctly attributed to 'Sébastian Lecocq / Alamy Stock Photo' in all versions of the Research Highlight.

Published online: 2 November 2017

https://doi.org/10.1038/s41550-017-0303-1 